\title{
SƯ DỤNG PHÂN TÍCH HƯớNG ĐỐI TƯợNG ĐỂ PHÁT TRIỄN NHỮNG HỆ THỐNG LỚN TÍCH HợP
}

\author{
ĐOÀN VĂN BAN
}

\begin{abstract}
This paper will discuss an oject-oriented analysis used in the development of integrated information systems. A system can be viewed as a collection of entities that interact together to accomplish certain objectives. In object-oriented analysis, the entities are called the objects. Object-oriented analysis refers to the methods of specifying of the software in terms of real-world objects, their behaviour, and their interactions. Objectoriented design on the other hand, turns the software requirements into specifications for objects and derives class hierarchies from which the objects can be created. The steps of object-oriented system development are described here. After that, we present a prototype simulating telecommunication services based on the use of object-oriented techniques. The general object model designed to process the telecommunication services is introduced and explained.
\end{abstract}

\section{GIỚI THIỆU}

Mực đích của phân tích hướng đối tượng là xác định đầy đủ, chính xác các lớp đối tượng của hệ thống bao gồm cà dữ liệu lẫn những phương thức (hàm thành phần) mô tả hành vi và mối quan hệ của chúng trong hệ thống. Phương pháp hướng đối tượng nhằm thực hiện kết hợp những thành phần dữ liệu với các phương thức xử lý (quá trình) để tạo ra những cấu trúc mô tả chính xác mọi hành vi của các thực thể trong hệ thống. Điều này trái ngược với phương pháp phân tích có cấu trúc mà theo đó việc phân tích dữ liệu và các quá trình thường là tách rời nhau [8]. Trong phân tích hướng đối tượng, quá trình phải được liên kết chặt chẽ với lớp chứa những hàm tương ứng với quá trình đó.

Một trong những ưu điểm chính của phương pháp hướng đối tượng là khả năng tạo ra những công cụ mạnh giúp chúng ta có thể phát triển được những hệ thống phần mềm có tính "mở", có tính "sử dụng lại" cao, an toàn thông tin, ... $[2,3]$. Đặc tính chính giúp chúng ta thực hiện được điều đó chính là cơ chế kế thừa. Trong thiết kế, khái niệm kế thừa cho phép chúng ta định nghĩa những lớp mới trên cơ sở mở rộng các lớp đối tượng đã được thiết kế tốt, bổ sung thêm một số thuộc tính, hàm thành phần mô tả chi tiết hơn về những đố tường cụ thễ (nguyên lý chi tiết hóa) hoặc từ một nhóm các lớp có một số đặc tính giống nhau, 
gộp chung những tính chất đó để tạo ra lớp mới, gọi là lớp trừu tượng (nguyên lý tổng quát hóa). Cả hai cách tiếp cận đó đều tạo ra cấu trúc phân cấp các lớp đối tượng theo quan hệ kế thừa $[1,2,5]$. Quan hệ kế thừa là quan hệ có tính bắc cầu nên các lớp con cháu (lớp kế thừa) sử dụng lại những đặc tính mà những lớp cơ sở (những lớp cha mẹ ở mức cao hơn trong cấu trúc phân cấp) đã có và được mở rộng, bổ sung thêm những đặc tính mới để mô tả được đúng, chính xác từng đối tượng trong hệ thống cũng như tạo ta khả năng cho hệ thống phần mềm có tính mở. Trừu tượng hóa dữ liệu là cách biểu diễn các nhóm đối tượng có liên quan bằng một lớp chứa những đặc tính chung nhất của chúng và bó đi những tính chất khác. Nhiều đặc tính của hướng đới tượng được xây dựng trên cơ sở khái niệm trừu tượng hóa, khái quát hóa. Đó là những nguyên lý cơ bản trong xây dựng các đặc tả và mô hình cấu trúc cho hệ thống.

Đề phát triển được hệ thống phần mềm hướng đối tượng thì trước tiện phải xác định danh sách các đối tượng, sau đó là mối quan hệ tương tác của chúng trong hệ thống. Bản thân các đối tượng chưa đặc tả được đầy đủ về hệ thống. Hệ thống phải là một kết hợp của nhiều đối tượng có quan hệ chặt chẽ với nhau và trao đổi với nha bằng các "thông báo". Điều quan trọng trong phương pháp hướng đối tượng là đặc tả các lớp đối tượng (có thể sư dụng $\mathrm{C}++$ là ngôn ngữ hướng đối tượng hỗ trợ cho việc mô tả khái niệm kế thừa và sử dụng lại [4] hoặc sử dụng ngôn ngữ đặc tả RAISE [7]) sao cho nhữg khái niệm được xây dựng không quá gắn chặt vào một hệ thống cụ thể và chúng có thể dễ dàng sử dụng lại trong những hệ thống khác, đồng thời xóa bỏ được những hố ngăn cách giữa các bước phân tích, thiết kế và lập trình trong quá trình phát triển những hệ thống phần mềm, nhất là đối với hệ thống thông tin tích hợp cỡ lớn.

Trong bài này chúng tôi đề cập đến việc sử dụng phân tích hướng đối tượng để xây dựng những hệ thống thông tin lớn tích hợp. Sau khi nêu những bước cần thực hiện để phát triển những hệ thống hướng đối tượng, chúng tôi phân tích các lớp đối tượng trong hệ thống dịch vụ thông tin để xây dựng mô hình cấu trúc cho hệ thống thông tin viền thông.

\section{SƠ ĐỒ KHÁI NIÊMM - KHUNG NHÌN CẤU TRÚC}

Sơ đồ khái niệm cũng giống như sơ đồ quan hệ thực thể. Nhưng thay vì đặc tả các thực thễ thông qua các thuộc tính và quan hệ giữa chúng, sơ đồ khái niệm tập trung chính vào các lớp đối tượng và mối quan hệ của chúng trong hệ thống. Sơ đồ khái niệm là công cụ tốt để mô tả cấu trúc của hệ thống trong phân tích hướng đối tượng.

Trong sơ đồ khái niệm, lớp được sử dụng để phân loại các thực thể trong thế giới thực. Thực thể có thể ở dạng vật lý như người, vật hoặc là những khái niệm như "Dịch_vụ" mà chúng tham gia vào một số hoạt dộng nhất định trong hệ 
thống. Lớp trong sơ đồ khái niệm tương đương với khái niệm thực thễ trong sơ đồ quan hệ thực thễ. Một lớp lại có thể phân chia thành một lớp con khác nhau. Những lớp con có thể kế thừa được những thuộc tính (dữ liệu) và các phương thức xử lý (hàm thành phần) của các lớp cơ sở.

Sơ đồ khái niệm xác định cấu trúc để duy trì dữ liệu và các phương thức cho bước cài đặt hướng đối tượng. Phương thức được sử dụng để tạo lập hay kết thúc hoạt động cừa các đối tượng, thay đồi, cập nhật những yêu cầu cần xử lý của chúng.

\section{MÔ HÌNH NGŨ̃ NGHĨA - KHUNG NHÌN VỀ HÀNH VI CỦA HỆ THỐNG}

Mô hình hướng đối tượng dựa trên cách tiếp cận phù hợp cho việc thiết kế, xây dựng các đơn thể của hệ thống. Hệ thống hướng đối tượng gắn chặt dữ liệu với các phương thức mô tả hành vi của đối tượng trong sự cố kết tồng thể của hệ thống. Cách tiếp cận này rất phù hợp để phát triển những hệ thống phức tạp như hệ thống giao diện đồ họa với người sử dụng (Graphic User Interface - GUI) đặc biệt là những hệ thống thông tin lớn tích hợp như hệ thống thông tin viễn thông mà chúng ta sẽ đề cập đến ở phần sau. Hệ thống dược điều khiển thông qua các sự kiện. Sự kiện là sự thay đổi trạng thái của đối tượng như khởi tạo, kết thúc hay phân chia lại các lớp đối tượng v.v... Như vậy sự kiện biểu diễn cho sự biến đổi trạng thái được thực hiện thông qua các bộ xứ lý và chúng điều khiển mọi hoạt động của hệ thống. Giữa bộ xử lý hành động và bộ xử lý quyết định có sự phân biệt:

- Bộ xử lý hành động luôn luôn thực hiện các phép biến đổi trạng thái và sự thay đổi đó khá phức tạp.

- Bộ xử lý quyết định xác định trạng thái hiện thời của đối tượng dựa trên giá trị tính được của biểu thức điều kiện (biểu thức Boolean). Nếu điều kiện thỏa (biểu thức Boolean có giá trị "true") thì sự kiện tương ứng sẽ xuất hiện và trên các đối tượng đó xuất hiện những thay đổi cần thiết. Bộ xử lý quyết định thường sử dụng đồng hồ (clock) để đồng bộ hóa hoạt động trong hệ thống.

Một điểm quan trọng cần lưu ý là giữa các bộ xử lý chỉ có sự liên kết lỏng nên chúng không biết được những xử lý khác làm việc như thế nào. Bộ xử lý quyết định tính giá trị của hàm Boolean để xác định trạng thái của đối tượng là độc lập với những điều kiền gây ra sự biến đổi đó (nguyên lý này ngược lại với nguyên lý "token" trong mạng Petri).

Tóm lại: nhiệm vụ của phân tích, thiết kế hướng đối tượng là trên cơ sở phân tích các yêu cầu của người sử dụng và tìm hiều những hệ thống thực tế liên quan để xác định được đầy đủ và chính xác các lớp đối tượng trong không 
gian bài toán sau đó chuyển chúng sang không gian lời giải, xây dựng mô hình kiến trúc và mô hình tính toán cho hệ thống phần mềm. Điều quan trọng là cần phải tạo ra được cấu trúc phân cấp cho các lớp đối tượng dựa trên các quan hệ kế thừa và sử dụng lại. Để xây dựng hệ thống phần mềm hướng đối tượng nhất là những hệ thống thông tin lớn tích thì cần thực hiện những bước sau:

1. Hệ thống được xem như là tập những đối tượng tương tác với nhau thông qua việc trao đổi các thông báo nên nhiệm vụ đầu tiên của người phát triển hệ thống là xác định đầy đủ, chính xác các lớp đối tượng và những thành phần cơ bản của chúng. Danh sách các đối tượng có thể định được dựa trên văn bản mô tả bài toán (đối tượng thường là các danh từ) kết hợp với những kinh nghiệm của người phân tích và từ danh sách những đối tượng đã được xây dựng những hệ thống tương tự trong thực tế.

2. Phân tích mối quan hệ giữa các lớp, khả năng sứ dụng lại, kế thừa và bao hàm của dữ liệu cũng như hàm thành phần xứ lý trên những dữ liệu đó để đưa ra mô hình cấu trúc phân cấp giữa các lớp theo nguyên tắc tồng quát hóa (trừu tượng hóa dữ liệu) giúp cho việc có thể tạo ra được những hệ thống mở, có khả năng sử dụng lại ở mức cao.

3. Lựa chọn ngôn ngữ đặc tả phù hợp (như RAISE, hoặc $\mathrm{C}++$ ) để đặc tả các lớp đối tượng bao gồm cả các dữ liệu và các hàm thành phần nhằm xóa bỏ hố ngăn cách giữa các bước trong quá trình phát triển hệ thống và hỗ trợ cho việc thực hiện cài đặt được dễ dàng hơn.

4. Thiết kế các lớp đối tượng và mối quan hệ của chúng trong hệ thống. Các lớp đối tượng chỉ trao đổi được với nhau thông qua các thông báo, nghĩa là chúng chỉ có thể truy nhập đến dữ liệu của đối tượng khác thông qua những giao diện đó là những hàm thành phần. Ngoài những hàm thể hiện những đặc tính cơ bản của đối tượng trong lớp, chúng ta cần bồ sung thêm:

- Những hàm quản lý lớp như tạo lập, hủy bỏ các đối tượng dưới dạng các cấu tử (constructor) hoặc hủy từ (destructor);

- Những hàm để cập nhật dữ liệu và hiền thị thông tin về đối tượng;

- Các hàm xử lý lỗi trong quá trình tính toán, trao đối thông tin của các đối tượng trong hệ thống và với những hệ thống khác.

5. Xây dựng các hàm thành phần. Mỗi đối tượng cần phải thực hiện một số chức năng nào đó và hành vi của chúng được thể hiện thông qua các hàm thành phần. Cũng giống như phương pháp thiết kế có cấu trúc, chúng ta có thể sứ dụng kỹ thuật phân rã chức năng theo cách tiếp cận trên - xuống (top - down) và phương pháp làm mịn dần để thiết kế các hàm thành phần của từng lớp đối tượng.

6. Bước cuối cùng trong khâu thiết kế hệ thống là xây dựng chương trình chính. Nhiệm vụ của chương trình chính là: 
- Nhập dữ liệu, thông tin các loại: âm thanh, hình ảnh tĩnh hay động từ nhiều nguồn khác nhau (từ người sử dụng, cơ sở dữ liệu, hay từ nhiều hệ thống khác v.v...) bằng nhiều phương pháp khác nhau.

- Tạo ra các đối tượng theo định nghĩa của các lớp đã được thiết kế tốt.

- Tồ chức thực hiện trao đổi thông tin giữa các đối tượng trong hệ thống và với những hệ thống khác.

- Lập các báo cáo hay hiển thị những kết quả xử lý trên màn hình, máy in hoặc lưu trữ, bảo quản thông tin theo yêu cầu người sử dụng trên nhiều loại phương tiện khác nhau.

\section{XÂY DỰNG HỆ THỐNG THÔNG TIN VIỄN THÔNG}

Hệ thống thông tin viễn thông không chỉ giới hạn phục vụ cho hệ thống điện thoại truyền tin mà còn có khả năng thực hiện những dịch vụ cao cấp khác như hội nghị, đa phương tiện kết hợp nghe nnìn (videotelephony) và nhiều dạng dữ liệu, hay thông tin khác. Trong mỗi cuộc gọi, người sử dụng có thể ấn định một số phương thức phục vụ tương ứng với loại thông tin và phương tiện mà họ mong muốn. Chính điều này làm tăng độ phức tạp của hệ thống phần mềm.

Cách tiếp cận hướng đối tượng cung cấp những phương pháp và công cự đủ mạnh, phù hợp để phát triển những hệ thống thông tin lớn tích hợp, đáp ứng được những yêu cầu trên và hỗ trợ để tạo ra hệ thống mớ, dễ dàng thay đổi theo yêu cầu. Trong phần này chúng ta sừ dụng phương pháp hướng đối tượng để xây dựng hệ thống thông tin viễn thông đáp ứng những yêu cầu như đã nêu ở trên.

\section{Những đăc tính chung}

Hệ thống thông tin viễn thông thực hiện những dịch vụ sau:

- Dịch vụ điện thoại.

- Dịch vụ đa phương tiện: videophony và những dạng dữ liệu, thông tin khác.

- Những dịch vụ khác:

+ Truyền tải các tính hiệu, thông tin trong khi vẫn thực hiện các dịch vụ gọi, truyền tin bình thường;

+ Dừng tạm thời một cuộc trao đổi thông tin trong hệ thống;

+ Thực hiện những cuộc gọi liên tiếp cho đến khi được trả lời;

+ Phục vụ hội nghị bằng da phương tiện video;

+ Phục vụ "hội đàm" máy tính, cho phép người sử dụng máy tính ở các đầu cuối tọa đàm với những trạm khác khi được phép.

Như vậy hệ thống phải thực hiện đa chức năng từ nhiều tổ hợp dịch vụ với nhiều dạng thông tin: âm thanh, dữ liệu, hình ảnh tĩnh, dộng cho nhiều ứng dụng khác nhau. 
Hệ thống thông tin viễn thông được tố chức theo kỹ thuật ATM phục vụ việc nối mạng theo mô hình OSI [6].

\section{Phân tích thiết kể các lớp đổi tượng cúa hệ thống thông tin viễn thông}

Theo quan điểm của người sử dụng, sự truyền tin trong hệ thống thông tin là phương tiện (quan hệ) cho phép gửi nhiều loại thông tin khác nhau: âm thanh, hình ảnh, dữ kiệu, văn bản, v.v... tới các đối tác ở những khoảng cách tùy ý. Phân tích hệ thống bưu điện truyền thống, kết hợp với những kinh nghiệm và các hồ sơ tài liệu về các hệ thống truyền tin, chúng ta xây dựng được danh sách các lớp đối tượng chính bao gồm: Terminal, Thuê_bao, Dịch_vụ, Truyền_tin, Trạm_trung_chuyển (kết nối gữa các trạm với nhau), Kế_nối_mạng. Các đối tượng này quan hệ với nhau theo mỗi quan hệ Khách_hàng/Người_phục_vụ (Client/Server). Mô hình truyền tin của hệ thống được mô tả như sau:

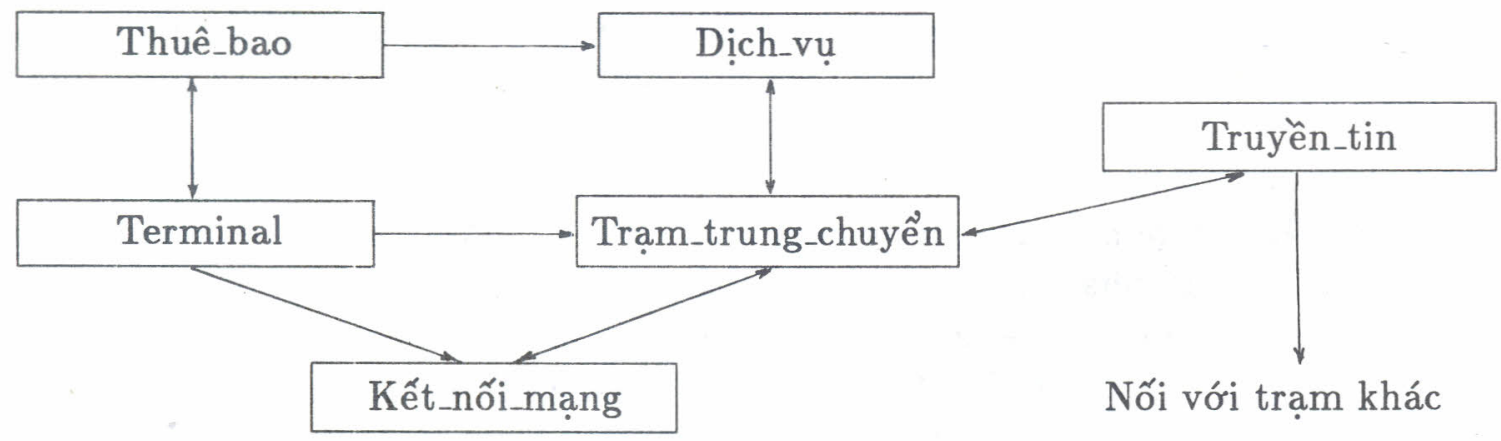

Hình 1. Sơ đồ khái niệm của hệ thống thông tin viễn thông

Trong mũi tên $\longrightarrow$ chỉ mối quan hệ Khách_hàng/Người_phục_vụ giữa các lớp đối tượng còn $\longleftrightarrow$ mô tả quan hệ hai chiều: một đối tượng có thể vừa là Khách_hàng vừa là Người_phục_vụ của một đối tượng khác.

Một trong những đặc trưng quan trọng nhất (và cũng là ưu điểm nổi bật) của phương pháp hướng đối tượng là hỗ trợ để tạo ra các lớp đối tượng trên cơ sở kế thừa từ những lớp đã được xây dựng trước (gọi là lớp cơ sở). Các lớp này sử dụng hầu như tất cá những thành phần của lớp cơ sở (trừ những dữ liệu, hàm thành phần khai báo ở vùng sở hữu riêng không cho phép kế thừa) và được bỗ sung thêm những đặc tính (dữ liệu, hàm mới) để mô tả cho những đối tượng được quyền sử dụng một số tính chất của các đối tượng của các lớp khác. Nguyên lý kế thừa hỗ trợ cho việc tạo ra cấu trúc phân cấp các lớp đối tượng. Chúng ta hãy phân tích lớp Dịch_vụ, một thành phần quan trọng nhất của mô hình nêu trên. hệ thống thông tin viễn thông hiện đại phải thực hiện được hai dịch vụ: dịch vụ bưu điện truyền thống (Dịch_vụ_BĐ) và những dịch vụ cao cấp khác (Dịch_vụ_khác). Lớp Dịch_vụ_BĐ lại có thể chia thành những lớp con kế 
thừa như: điện thoại (Phone), những dịch vụ đa phương tiện (multi_media) còn lớp Dịch_vụ_khác lại có thể chia thành những lớp con kế thừa như: phục vụ đàm thoại máy tính (Đàm_thoại_MT), phục vụ hội nghị (Hội_nghị), đặt gọi (Đặt_gọi), thuê riêng kênh truyền (Thuê_kênh), v.v... Tiếp tục phân tích các đối tượng (các thực thể) trong hệ thống và mối quan hệ (kế thừa mô tả bằng ) giữa chúng ta có được sơ đồ phân cấp như sau:

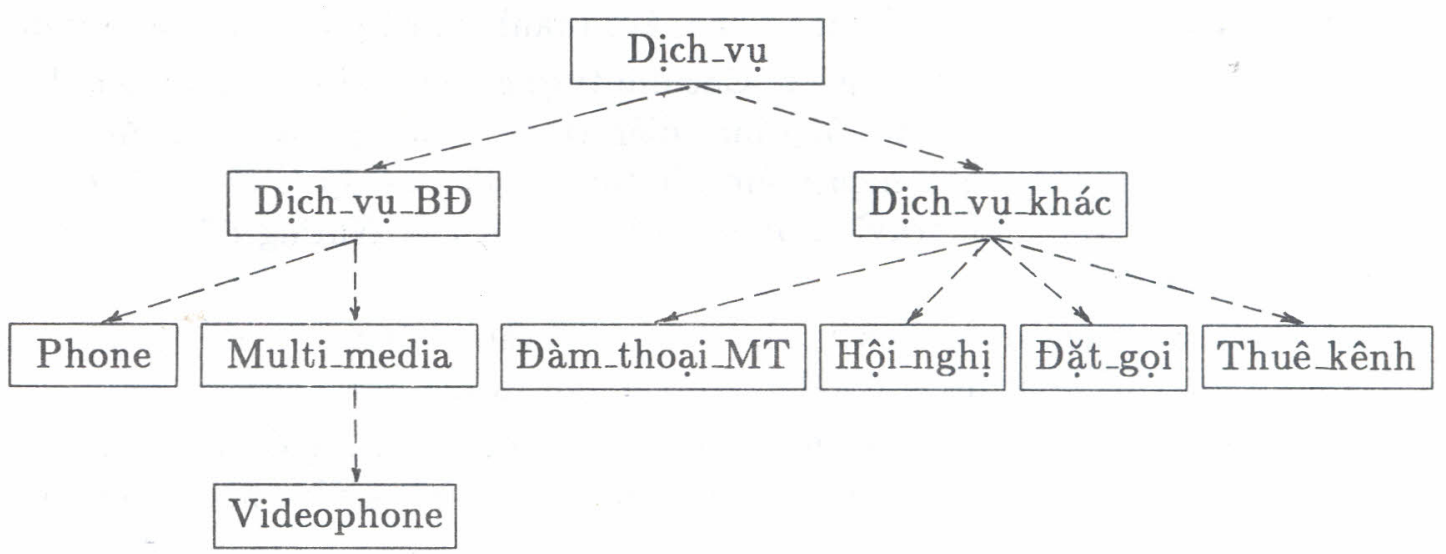

Hình 2. Những lớp đối tượng kế thừa của lớp Dịch_vụ

Khả năng sử dụng lại là đặc tính quan trọng của lập trình hướng đối tượng. Việc sử dụng lại những đ̛ơn thể chương trình, những lớp đã thiết kế tốt, đã được kiểm nghiệm không những tiết kiệm được nhiều thời gian, công sức mà còn làm tăng khả năng tương thích và độ tin cậy của hệ thống. Ngôn ngữ đặc tả hệ thống như RAISE rất tiện lợi cho việc đặc tả những hệ thống lớn thích hợp như hệ thống thông tin viễn thông. $\mathrm{C}++$ cũng hố trợ rất mạnh cho việc đặc tả những khái niệm mới của phương pháp hướng đối tượng như: kế thừa, tương ứng bội, v.v... Nhiều lớp đối tượng được thiết kế trong $\mathrm{C}++$ có thể sứ dụng lại theo nhiều cách khác nhau. Khi một lớp đã được định nghĩa thì người lập trình có thể sứ dụng nó trong những thiết kế riêng của mình. Những lớp đó là cơ sở để tạo ra các lớp mới trên cơ sở sứ dụng lại những đặc tính quan trọng và cơ bản của các lớp trước (bạn đọc quan tâm có thể tham khảo [2] - từ trang 161 đến 180 về đặc tá các cơ chế thừa trong $\mathrm{C}++$ ). 'Lớp Dịch_vụ ở hình 2 được thiết kế bao gồm những đặc tính chung nhất (dữ liệu và những hàm thành phần mô tả hành vì cho tất cá các dịch vụ trong hệ thống) như: các giao thức (protocols), các mã thông tin, v.v... Lớp Dịch_vụ_BĐ và Dịch_vụ_khác cùng kế thừa từ lớp Dịch_vụ, nên ngoài những thành phần được kế thừa từ Dịch_vụ, mỗi lớp lại được bổ sung thêm những đặc tính mới để thực hiện được những chức năng của chúng.

Tất cả các đối tượng trong lớp Dịch_vụ_BĐ đều được thực hiện một số nhiệm vụ để có hành vi chung: 
- Để thực hiện được dịch vụ trao đổi thông tin thì cần phải: kiểm tra các phương tiện sẵn sàng truyền tin, cấp phát tài nguyên, xác định đối tác, truyền các yêu cầu tới đối tác, v.v.

- Giải phóng các tài nguyên mỗi khi kết thúc một dịch vụ.

Mỗi dịch vụ (Phone, Videophone) lại có những đặc tính riêng của chúng. Ví dụ Vodeophone thực hiện với đa phương tiện (multimedia) nên nó đòi hỏi phải có các thành phần xử lý dữ liệu về thông báo, âm thanh và cả phần hình ảnh video.

Lớp Dịch_vụ_khác cũng tương tự. Các đối tượng của nó phải thực hiện được những chức năng chính của hệ thống bưu điện truyền thông. Sau đó chúng phải có những thành phần để thực hiện thêm các thành phần bỗ sung cho việc truyền tín hiệu, thuê trọn gói kênh truyền, phục vụ hội nghị, v.v. Những dối tượng này có các đặc tính chung:

- Sau khi thực hiện xong dịch vụ bưu điện truyền thống, các đối tượng cúa Dịch_vụ_khác sẽ hoạt động độc lập với các đối tượng cúa Dịch_vụ_BĐ.

- Bất kỳ lúc nào chúng cũng có thể (hoặc không thể) sử dụng dịch vụ bưu điện truyền thống, ví dụ Hội nghị có thể sử dụng Phone bất kỳ lúc nào cũng được.

Phân tích mối quan hệ giữa các lớp (các thực thể) trong hệ thống đễ đưa ra cấu trúc phân cấp theo quan hệ kế thừa là cơ sở để tạo ra những hệ thống có tính mở và có khà năng sử dụng lại ở mức cao.

\section{Mô hình truyền tin: mô hình ngữ nghĩa}

Dựa vào nhiệm vụ và chức năng cúa các lớp đối tượng trong hệ thống, chúng ta có thể phân loại chúng thành 3 nhóm:

1) Những lớp mô tả thực thể của hệ thống vật lý như: Thuê_bao, Terminal, v.v., chứa tất cả những thông tin cần thiết cho các dịch vụ của hệ thống;

2) Các đối tượng dịch vụ: Phone, Videophone, và các dịch vụ khác, chứa tất cả những mô tả toàn cục (lớp trừu tượng) hoặc mô tả chi tiểt cho từng đối tượng cụ thể (Phone, Vodeophone, Hội_nghị, v.v.).

3) Những lớp thực hiện truyền tin như: Kết_nối_mạng, Trung_chuyển, Truyền_tin, làm nhiệm vụ kết nối các đầu cuối theo yêu cầu.

Tất cà các đối tượng trong hệ thống trao đổi với nhau thông qua các thông báo. Có hai loại thông báo:

- Những thông báo liên quan đến việc kết nối giữa các điểm trong hệ thống (nối kênh/giải phóng) và kết quả là thực hiện các giao thức kết nối (association protocol).

- Những thông báo liên quan đến những dịch vụ và kết quả là thực hiện các giao thức dịch vụ (service Protocol).

Các giao thức dịch vụ đề cập đến những dịch vụ về quản lý: mở/đóng một cuộc tọa đàm, dịch vụ đàm thoại: nối kênh truyền, truyền tin, cấp phát/giải 
phóng các phương tiện phục vụ đa phương tiện (audio, video, và dữ liệu) và nhiều dịch vụ khác.

Tóm lại, hệ thống thông tin viển thông (theo hướng đối tượng) có thể xem là hệ thống trao đổi giữa $n$ người sử dụng, bao gồm tập $n$ kết nối với $n$ trạm cuối được mô tả như ở hình 3 .

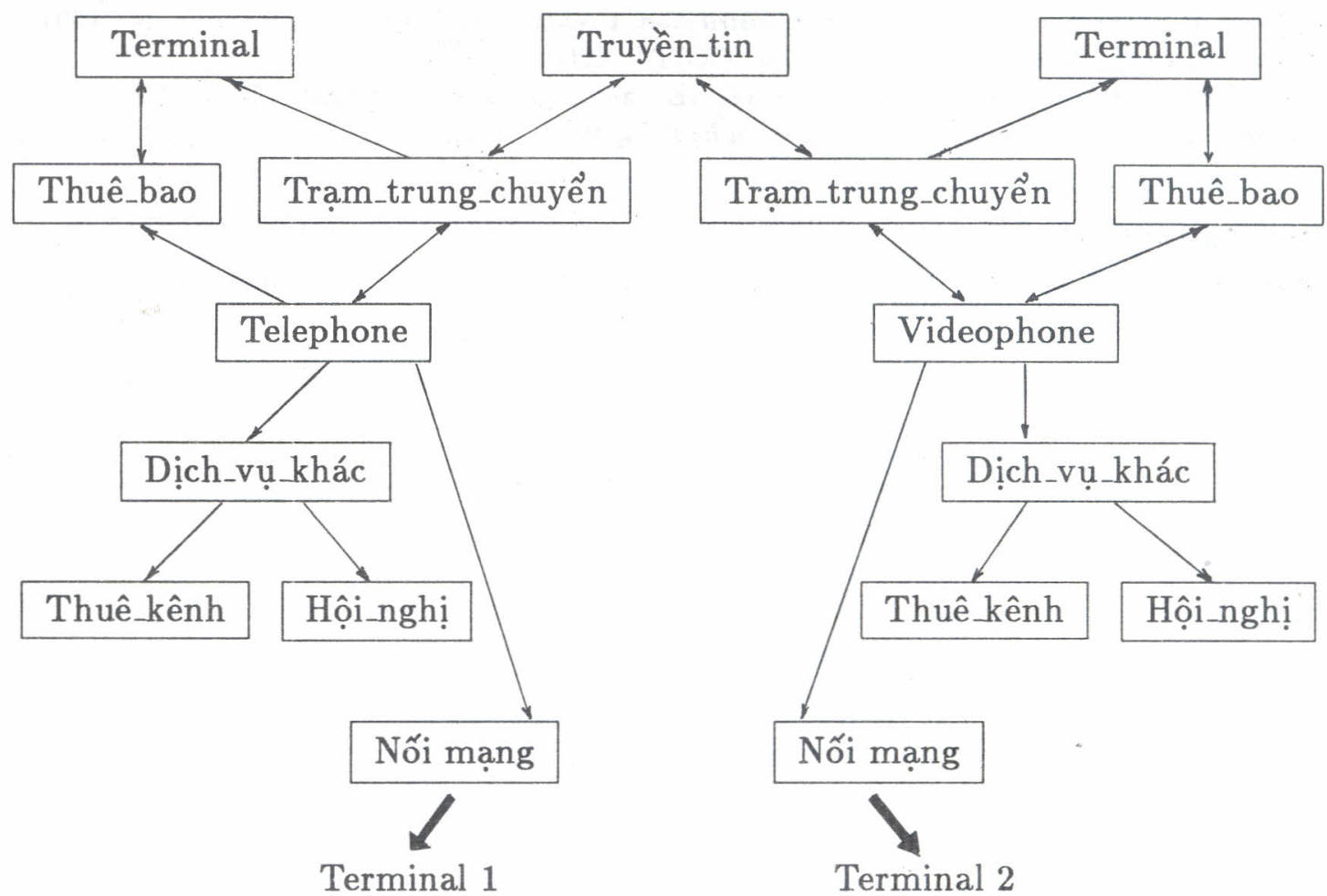

Hinh 3. Mô hình ngữ nghĩa: Mối quan hệ giữa các lớp tham gia thực hiện cuộc đàm thoại giữa hai trạm thuê bao của hệ thống truyền tin

\section{KẾT LUẬN}

Mô hình hướng đối tượng xem hệ thống là một tập các đối tượng tương tác với nhau để thực hiện các mục tiêu nhất định. Cách tiếp cận này rất phù hợp với cách quan sát của chúng ta về thế giới thực và tạo ra những công cự mới, hữu hiệu để phát triển những hệ thống có tính mở, dễ thay đổi theo yêu cầu của người sử dụng, có khả năng sử dụng lại những thành phần đã được thiết kế, lập trình tốt, v.v..., đáp ứng được các tiêu chuẩn của phần mềm chất lượng cao theo yêu cầu của nền công nghệ thông tin hiện đại. Phân tích, thiết kế hướng đối tượng tó ra phù hợp để xây dựng những hệ thống thông tin lớn phức hợp, ví dụ như hệ thống thông tin viễn thông. 


\section{TÀI LIẾU THAM KHẢO}

1. Coad P. and Yurdon E., Object - Oriented Analysis, Yourdon Press and Prentice - Hall, 1979.

2. Đoàn Văn Ban, Phân tích, thiết kế và lập trình huớng đối tượng, Báo cáo đề tài Trung tâm KHTN \& CNQG, Hà Nội, 1996.

3. Graham I., Object - Oriented Methods, Affism - Wesley, 1992.

4. Hendenson P., Oject - Oriented Specification and Design with $C++$, McGraw-Hill, 1993.

5. Meyer B., Object - Oriented Software Construction, Prentice-Hall Englewood Cliffs, NJ 1991.

6. Open System Interconnexion ISO 7498/CCITT X200.

7. The RAISE Specification Language, The RAISE Language Group, Prentice-Hall, 1992.

8. Viện Tin học, Phân tích, thiết kê và cài đặt hệ thống thông tin quán lý, Dự án VIE/88/035, 1991.

Viện Công nghê thông tin

Trung tâm KHTN và CNQG

Nhận bài ngày 16-8-1997 